\title{
Efficacy and tolerability of pregabalin as preventive treatment for migraine: a 3-month follow-up study
}

\author{
Raffaella Pizzolato • Veronica Villani • \\ Luca Prosperini · Alessandro Ciuffoli • \\ Giuliano Sette
}

Received: 5 January 2011/Accepted: 23 March 2011/Published online: 9 April 2011

(C) The Author(s) 2011. This article is published with open access at Springerlink.com

\begin{abstract}
Migraine is a common neurological disorder and epidemiological studies have documented its high social and economic impact. Unfortunately, preventive treatment is often insufficient to substantially reduce migraine frequency or it is not well tolerated. Antiepileptic drugs are increasingly used in migraine prevention. However, data on efficacy and tolerability of pregabalin in patients with migraine are still lacking. Our aim was to evaluate efficacy and tolerability of pregabalin in patients with migraine. We recruited 47 patients who started pregabalin at $75 \mathrm{mg} /$ day, which was titrated to $300 \mathrm{mg} /$ day as tolerated. A total of six patients (13\%) reported one or more side effects during the intake of pregabalin; however, three of them discontinued pregabalin, because side effects were intolerable and persistent. Statistically significant reduction in migraine frequency compared to baseline $(p<0.001)$ was evident after 1 and 3 months of treatment. A greater frequency reduction was observed in those patients who increased the dosage within the first month of therapy. Our data suggest that pregabalin may be well tolerated and may represent an alternative preventive treatment in migraneurs. Limitations of the present study were a small sample size and an uncontrolled, open-label design; further randomized case-control studies are warranted to confirm our findings.
\end{abstract}

R. Pizzolato · V. Villani · A. Ciuffoli · G. Sette

Neurological Headache Centre, S. Andrea Hospital,

Sapienza University, Rome, Italy

V. Villani $(\bowtie) \cdot$ L. Prosperini $\cdot$ A. Ciuffoli

Department of Neurology and Psychiatry, Headache Centre,

S. Andrea Hospital, Sapienza University,

Via di Grottarossa, 1035, 00189 Rome, Italy

e-mail: veronicavillani@hotmail.it
Keywords Migraine P Pregabalin .

Prophylaxis therapy $\cdot$ Disability

\section{Introduction}

Migraine is a common neurological disorder that affects approximately $12 \%$ of adult population [1]. Epidemiological studies have documented a high impact and the profound effect that the disorder can have on an individual's quality of life [2]. Although migraine is a highly prevalent and disabling condition, it is underdiagnosed and undertreated [3, 4]. Recent advances have been made in the management of migraine, but pharmacologic treatment options are still far from optimum, leaving many patients without pain-free treatments or with unpleasant side effects $[5,6]$. Nowadays, antiepileptic drugs (AEDs) are increasingly used in migraine treatment $[7,8]$; in general, these agents are considered both effective in reducing attack frequency and reasonably well tolerated, although few robust trials are available for AEDs other than divalproex sodium and topiramate $[9,10]$. These are favored by Level 1 evidence and clinical experiences as first-line preventive drugs for migraine [10, 11]. Recently, other different AEDs such as pregabalin are emerging in the prophylaxis of migraine $[12,13]$.

Pregabalin is currently recommended for the treatment of partial seizures, pain associated with post-herpetic neuralgia, diabetic neuropathy, fibromyalgia and spinal cord damage, as well as in the treatment of generalized anxiety disorder [14-16]. Pregabalin, through binding to the alpha2-delta subunits of hyperexcited, voltage-gated calcium channels, reduces the calcium influx at neurons terminals and subsequently reduces the synaptic release of several excitatory neurotransmitters such as glutamate, 
noradrenaline and substance $\mathrm{P}$. Therefore, pregabalin restores the hyperexcited calcium channels to a normal state [17]. Pregabalin mechanism of action is coherent with the available data concerning glutamatergic mechanism in migraine physiopathology.

The primary aim of this study was to evaluate the effectiveness of pregabalin in reducing migraine frequency; secondarily, we aimed to investigate the safety and the tolerability of pregabalin.

\section{Methods}

Outpatients with a diagnosis of migraine according to International Headache Criteria (ICHD II) [18], and regularly attending the Neurological Headache Centre of $\mathrm{S}$. Andrea Hospital in Rome were considered during a period of 6 months.

Those patients eligible for prophylactic treatment (i.e., patients with $\geq 4$ attacks per month) were asked to participate to this independent, uncontrolled, open-label, observational, prospective study. The exclusion criteria included an age younger than 18 years, medical or neurological disorders capable of causing headache, pregnancy, breastfeeding, and rescue medications overuse. An informed consent was obtained from each participant.

Following a 6-month period of wash-out after the last assumption of preventive agents, each eligible patient started pregabalin on a $75 \mathrm{mg} /$ day dosage, which was titrated up to a maximum of $300 \mathrm{mg} /$ day with a $75 \mathrm{mg}$ increase per week, as tolerated.

Patients were allowed to use acute pain medications during the evaluation period, including triptans, non-steroidal anti-inflammatory drugs, and antiemetics, but not other preventive therapies. They were allowed to take only 15 acute pain medications per months to avoid medication overuse.

Clinical data and the frequency of migraine (measured as days with headache per month) were collected for a 3-month period before starting the therapy, and after 1 and 3 months of pregabalin treatment, by means of patient diaries or during the 1-month scheduled interviews. The occurrence of side effects or adverse events were also recorded at first and third month of pregabalin intake.

\section{Statistical analysis}

To evaluate the efficacy of pregabalin, we performed an intention-to-treat analysis. All values are expressed as a mean \pm standard deviation $( \pm \mathrm{SD})$, or interval, as appropriate. Statistical differences over time in days with headache per month were analyzed using the univariate analysis of the variance (ANOVA) for repeated measures. Response rate in terms of proportion of patients with a $50 \%$ frequency reduction was also presented.

All $p$ values less than 0.05 (two-sided) were considered as significant. Statistical analyses were carried out by using a PC version of the Statistical Package for Social Sciences 16.0 (SPSS, Chicago, IL, USA).

\section{Results}

Forty-seven patients (10 males, 37 females) were included in the present study. The mean age at the study enrolment was $48.0 \pm 15.8$ years (interval 20-83 years). According to the ICHD-II criteria [18], 21 patients (45\%) had a diagnosis of migraine without aura and 26 patients $(55 \%)$ had a chronic migraine (i.e., patients with $\geq 15$ days with headache per month) without overuse of symptomatic drugs. Seven (15\%) patients (six chronic and one episodic migraneurs) had a psychiatric comorbidity according to DSM-IV criteria (three affected by depression, four affected by anxiety).

Only seven patients (15\%) had never used prophylactic treatment, while forty $(85 \%)$ tried at least one drug for migraine prophylaxis (see Table 1) before starting the pregabalin therapy. The migraine preventive medication class most commonly used was tricyclic antidepressants (38 patients, 95\%), followed by anticonvulsants (34 patients, 85\%), calcium-channel blockers (18 patients, $45 \%$ ) and $\beta$-blockers (10 patients, $25 \%$ ).

All patients started the pregabalin therapy at a dosage of $75 \mathrm{mg} /$ day. The majority of patients $(n=33)$ had a dose increase within the first month of therapy: 30 patients achieved a daily dose of $150 \mathrm{mg}$, one patient $225 \mathrm{mg}$, and two patients $300 \mathrm{mg}$. These different dosages were achieved according to the pregabalin tolerability and the compliance of the patients. The majority of patients did not reach the maximum daily dosage $(300 \mathrm{mg}$ ) also because of the concerns regarding the potential side effects, or satisfactory response to lower dosages.

Eleven patients $(23 \%)$ discontinued the pregabalin therapy after a mean time of $1.2 \pm 0.4$ months because of

Table 1 Migraine prophylactic drugs used before pregabalin

\begin{tabular}{llcl}
\hline Medication class & Drugs & No. of patients & $\%$ \\
\hline Antiepileptics & Topiramate & 20 & 50 \\
& Gabapentin & 24 & 60 \\
& Valproic acid & 4 & 10 \\
$\beta$-blockers & Propanolol & 10 & 25 \\
Calcium-channel blockers & Flunarizine & 15 & 37.5 \\
& Cinnarizine & 4 & 10 \\
Antidepressants & Amitriptyline & 38 & 95 \\
\hline
\end{tabular}


Table 2 Side effects reported by patients treated with pregabalin $(n=6)$
All these six patients had previous exposure to prophylactic drugs

\begin{tabular}{|c|c|c|c|c|c|}
\hline Sex & Age & Diagnosis & $\begin{array}{l}\text { Comorbid psychiatric } \\
\text { disorders }\end{array}$ & $\begin{array}{l}\text { Maximum } \\
\text { dosage }\end{array}$ & Side effects \\
\hline $\mathrm{F}$ & 51 & Migraine without aura & $\mathrm{N}$ & 75 & $\begin{array}{l}\text { Drowsiness } \\
\text { Blurred vision }\end{array}$ \\
\hline $\mathrm{F}$ & 78 & Chronic migraine & $\mathrm{Y}$ & 150 & $\begin{array}{l}\text { Drowsiness } \\
\text { Confusion }\end{array}$ \\
\hline $\mathrm{F}$ & 51 & Chronic migraine & $\mathrm{N}$ & 150 & Drowsiness \\
\hline M & 51 & Chronic migraine & $\mathrm{Y}$ & 150 & Dizziness \\
\hline $\mathrm{F}$ & 37 & Migraine without aura & $\mathrm{N}$ & 150 & $\begin{array}{l}\text { Drowsiness } \\
\text { Fatigue }\end{array}$ \\
\hline $\mathrm{F}$ & 59 & Chronic migraine & $\mathrm{N}$ & 150 & $\begin{array}{l}\text { Drowsiness } \\
\text { Abdominal pain }\end{array}$ \\
\hline
\end{tabular}

lack of effectiveness (seven), occurrence of side effects (three) and economic reasons (one). No serious adverse events occurred during the period of observation. A total of six patients $(13 \%)$, all with a previous exposure to prophylactic drugs, reported one or more side effects during the intake of pregabalin; three of them had tolerable and transient side effects (see also Table 2). There was no relationship between the patients'dosage of pregabalin, or the time of titration, and the occurrence of side effects (data not shown).

A significant reduction in frequency $(p<0.001)$ was observed after 1 and 3 months of treatment compared to the baseline ( -32 and $-31 \%$, respectively) (Fig. 1). When compared to the baseline value, $12(26 \%)$ patients had a reduction equal or more than $50 \%$ in number of days with headache per month. Overall, 28 patients $(60 \%)$ had at least a 1/4 attack frequency decrease (Table 3).

The significant reduction of attack frequency per month was similar (about 33\%) between episodic (from $6.8 \pm 3.0$ to $4.8 \pm 3.5$ ) and chronic migraineurs (from $27.6 \pm 5.2$ to $18.7 \pm 9.7)$.

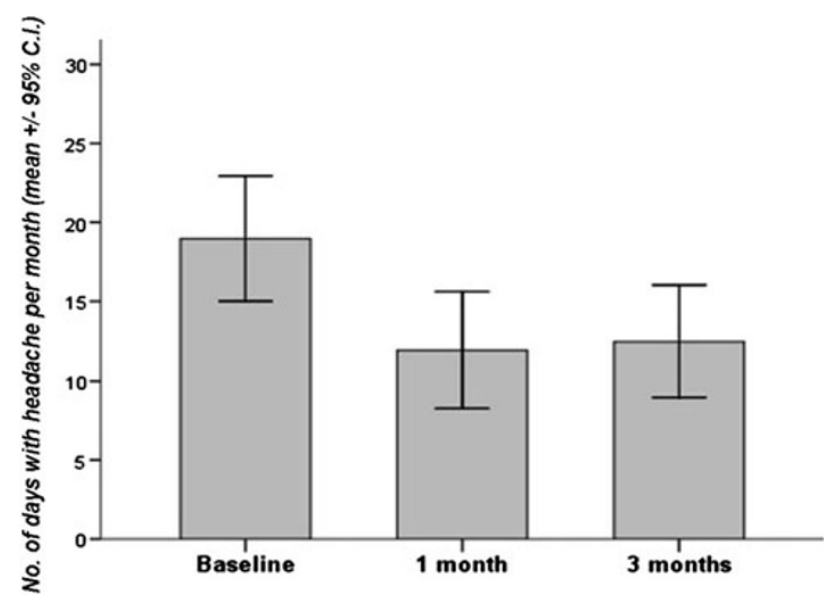

Fig. 1 Mean number of days with headache per month, with relative 95\% confidence intervals, at different time point for the whole study population. $p$ value $<0.001$ (ANOVA)
Table 3 Reduction in number of days with headache per month when compared to baseline value

\begin{tabular}{ll}
\hline Percentage of reduction & Patients, $n(\%)$ \\
\hline$\geq 50$ & $12(26)$ \\
$49-25$ & $16(34)$ \\
$<25$ & $19(40)$ \\
\hline
\end{tabular}

Furthermore, the level of response to pregabalin was not influenced by sex, age, previous use of prophylactic drug, or comorbid psychiatric disorders (data not shown), but a greater reduction in days with headache per month was observed in the 33 patients who reached a daily dose of at least $150 \mathrm{mg}$ (Fig. 2). We did not observe any significant reduction in migraine frequency at first month in patients receiving a daily dose of $75 \mathrm{mg}$. On the contrary, patients treated with a dose $\geq 150 \mathrm{mg} /$ day had beneficial effect even at first month, which was sustained at the end of the third month of therapy.

\section{Discussion}

We found a significant reduction in mean days with headache per month when compared to baseline after 1 and 3 months of therapy. Roughly, the one quarter of our study population had a response rate equal or more than $50 \%$ in terms of reduction of number of days with headache per month with respect to the baseline values. These findings are consistent with the modest, but clinically relevant improvement in attack frequency observed in another study of 30 chronic migraine subjects [12].

We observed a more relevant and faster reduction of attack frequency in patients who reached a daily dose of $150 \mathrm{mg}$ even in the first month. On the contrary, when the titration was slower, we found a delay in achieving a significant reduction in attack frequency, which was reached only at the third month of pregabalin therapy. 
Fig. 2 Reduction of mean number of days with headache per month with respect to the increase in the dose at the 1 -month visit. $p<0.001$ for patients who increased $(n=33)$ and $p=0.08$ for those who did not increase $(n=14)$ the pregabalin dose within the first month of treatment (ANOVA)


Increase of pregabalin dose within the $1^{\text {st }}$ month of treatment
Our results also suggest that pregabalin is safe (no serious adverse events occurred in our population) and generally well tolerated. In our sample the dose or titration of pregabalin did not seem to be related to the occurrence of side effects, which occurred in only six (13\%) patients assuming $\leq 150 \mathrm{mg} / \mathrm{day}$. Calandre et al. [12] reported that pregabalin is well tolerated, but $33.3 \%$ of patients exhibited dose-dependant side effects, especially after dosage adjustment; however, only two patients withdrew because of treatment-related adverse effects.

AEDs such as gabapentin and pregabalin may be better preventive therapies for old patients than the other therapeutic options, because of their low adverse effects on cardiovascular system and on mood disorders, such as anxiety and depression, that are very common in elderly. This explains the mean age range (20-83 years) of the sample studied higher than that of trials about other preventive therapies.

Being an observational study, our findings suffer from some limitations, including an uncontrolled and open-label design and the small sample size. Moreover, there was no evaluation of indirect measures regarding the efficacy of pregabalin, such as quantification of headache severity, or the number of rescue medications intake during the follow-up.

\section{Conclusion}

Despite limitations due to a small sample size and an uncontrolled, open-label design, the present study suggests that pregabalin may represent an efficacious and safe antimigraine agent in both episodic and chronic migraine patients.

\section{Conflict of interest None.}

Open Access This article is distributed under the terms of the Creative Commons Attribution License which permits any use, distribution and reproduction in any medium, provided the original author(s) and source are credited.

\section{References}

1. Lipton RB, Scher AI, Kolodner K et al (2002) Migraine in the United States. Epidemiology and patterns of health care use. Neurology 58(6):885-894

2. Leonardi M, Mathers C (2000) Global burden of migraine in the year 2000. Summary of methods and data sources. http://www. who.int/entity/healthinfo/statistics/bod_migraine.pdf. Accessed 5 Nov 2009

3. Stewart WF, Shechter A, Rasmussen BK (1994) Migraine prevalence. A review of population-based studies. Neurology 44(6 Suppl 4):S17-S23

4. Bigal ME, Lipton RB (2008) Clinical course in migraine: conceptualizing migraine transformation. Neurology 71(11):848-855

5. Bigal ME, Krymchantowski AV (2006) Emerging drugs for migraine prophylaxis and treatment. Med Gen Med 8(2):31

6. Ramadan NM (2007) Current trends in migraine prophylaxis. Headache 47(Suppl 1):S52-S57

7. Pappagallo M (2003) Newer antiepileptic drugs: possible uses in the treatment of neuropathic pain and migraine. Clin Ther 25(10):2506-2538

8. Buchanan TM, Ramadan NM (2006) Prophylactic pharmacotherapy for migraine headaches. Semin Neurol 26(2):188-198 
9. Ramadan NM (2006) Migraine headache prophylaxis: current options and advances on the horizon. Curr Neurol Neurosci Rep 6(2):95-99

10. Vikelis M, Rapoport AM (2010) Role of antiepileptic drugs as preventive agents for migraine. CNS Drugs 24(1):21-33

11. Chronicle E, Mulleners W (2004) Anticonvulsant drugs for migraine prophylaxis. Cochrane Database Syst Rev (3):CD003226

12. Calandre EP, Garcia-Leiva JM, Rico-Villademoros F, Vilchez JS, Rodriguez-Lopez CM (2010) Pregabalin in the treatment of chronic migraine: an open-label study. Clin Neuropharmacol 33(1):35-39

13. Masdrakis VG, Oulis P, Karakatsanis NA, Potagas C, Kouzoupis AV, Soldatos CR (2008) Remission of migraine attacks in a patient with depression who is taking pregabalin. Clin Neuropharmacol 31(4):238-240

14. Tassone DM, Boyce E, Guyer J, Nuzum D (2007) Pregabalin: a novel gamma-aminobutyric acid analogue in the treatment of neuropathic pain, partial-onset seizures, and anxiety disorders. Clin Ther 29(1):26-48

15. Arnold L, Mease P, Silverman S (2010) Pregabalin: an alpha2delta (alpha2-delta) ligand for the management of fibromyalgia. Am J Manag Care 16(5 Suppl):S138-S143

16. Teasell RW, Mehta S, Aubut JA, Foulon B, Wolfe DL, Hsieh JT, Townson AF, Short C, Spinal Cord Injury Rehabilitation Evidence Research Team (2010) A systematic review of pharmacologic treatments of pain after spinal cord injury. Arch Phys Med Rehabil 91(5):816-31

17. Ben-Menachem E (2004) Pregabalin pharmacology and its relevance to clinical practice. Epilepsia 45(Suppl 6):13-18

18. Headache Classification Committee of the International Headache Society. The International Classification of Headache Disorders, 2nd edn. Cephalalgia. 24(Suppl 1):1-160 